\title{
SAR evaluation in human body exposed to EM wave from NHA with metallic case
}

\author{
Shimpei Akimoto ${ }^{a}$, Satoru Kikuchi, Kazuyuki Saito, \\ Masaharu Takahashi, and Koichi Ito \\ Chiba University, \\ 1-33 Yayoi-cho, Inage-ku, Chiba 263-8522, Japan \\ a)s.akimoto@graduate.chiba-u.jp
}

Abstract: The portable radio terminal for business is usually hold in the vicinity of a human body, it is needed an attention for the specific absorption rate (SAR) in an abdomen. Therefore, in order to evaluate the SAR in the human body when wearing the portable radio terminals for business, the normal-mode helical antenna (NHA) with metallic case, which simulates the portable radio terminals for business at $150 \mathrm{MHz}$, was modeled. In addition, the calculations of SAR distributions employing an NHA with metallic case in the vicinity of the tissue-equivalent phantom were compared with the measurements. As a result, the SAR distributions of calculated result agree well with those of measured result.

Keywords: portable radio terminals for business, NHA, SAR, phantom

Classification: Microwave and millimeter wave devices, circuits, and systems

\section{References}

[1] M. Saito and M. Taki, "Biological effects of electromagnetic fields and health risks," J. IEICE, vol. 82, no. 6, pp. 572-579, June 1999 (in Japanese).

[2] Safety levels with respect to human exposure to radio frequency electromagnetic fields, $3 \mathrm{kHz}$ to $300 \mathrm{GHz}$, ANSI/IEEE Standard C95.1-2005, Oct. 2005.

[3] M. A. Jensen and Y. Rahmat-Samii, "EM interaction of handset antennas and a human in personal communications," Proc. IEEE, vol. 83, no. 1, pp. 7-17, Jan. 1995.

[4] A. D. Tinniswood, C. M. Furse, and O. P. Gandhi, "Computations of SAR distributions for two anatomically based models of the human head using CAD files of telephones and the parallelized FDTD code," IEEE Trans. Antenna Propag., vol. 46, no. 6, pp. 829-833, June 1998.

[5] J. Wang and O. Fujiwara, "FDTD analysis of dosimetry in human head model for a helical antenna portable telephone user," IEICE Trans. Commun., vol. E83-B, no. 3, pp. 549-554, March 2000.

[6] Y. Koyanagi, H. Kawai, K. Ogawa, and K. Ito, "Estimation of the local SAR in the human abdomen using a human body phantom and small an- 
tennas at $150 \mathrm{MHz}, "$ IEICE Trans. Commun., vol. J86-B, no. 7, pp. 12071218, July 2003 (in Japanese).

[7] Federal Communications Commission (FCC), "Evaluating compliance with FCC guidelines for human exposure to radiofrequency electromagnetic fields," OET Bulletin 65, Supplement C, Jan. 2001.

[8] N. Kuster and Q. Balzano, "Energy absorption mechanism by biological bodies in the near field of dipole antenna above $300 \mathrm{MHz}$," IEEE Trans. Veh. Tech., vol. 41, no. 1, pp. 17-23, Feb. 1992.

[9] IEC 62209-1 Human exposure to radio frequency fields from hand-held and body-mounted wireless communication devices Human models, instrumentation, and procedures Part 1: Procedure to determine the specific absorption rate (SAR) for hand-held devices used in close proximity to the ear (frequency range of $300 \mathrm{MHz}$ to $3 \mathrm{GHz}$ ), Feb. 2005 .

\section{Introduction}

The radio frequency $(\mathrm{RF})$ devices which are usually used in the vicinity of a human body have been increasing. Therefore, it is necessary to evaluate the interaction between the electromagnetic (EM) wave and the human, because it might be expected that human body is exposed to the EM waves radiated from the RF devices. Here, the influence of the EM waves on the human body is dependent on the frequency [1]. The EM waves mainly contribute the heat effect, which is generated by the absorption of the energy, above $100 \mathrm{kHz}$. The specific absorption rate (SAR) has been usually used for the primary dosimetric parameter of the EM wave exposure in the standards [2].

$$
\mathrm{SAR}=\frac{\sigma E^{2}}{\rho} \quad[\mathrm{W} / \mathrm{kg}]
$$

where, $\sigma$ is the conductivity of the tissue $[\mathrm{S} / \mathrm{m}], \rho$ is the density of the tissue $\left[\mathrm{kg} / \mathrm{m}^{3}\right]$, and $E$ is the electric-field strength (r. m. s.) inside the tissue $[\mathrm{V} / \mathrm{m}]$.

The SAR in the human body affected by EM wave from the cellular phones has been widely investigated $[3,4,5]$, because the cellular phones are used in the vicinity of the human head. However, the SAR of using the portable radio terminals for business has not been investigated so much. The EM waves of these devices penetrate to the deep region of the human body, because the wavelength of the EM waves of the device operating in VHF band are longer than those of a cellular phone operating in the $\mathrm{GHz}$ band. Therefore, it is necessary to evaluate the SAR of the human body, when they are wearing a portable radio terminal for business on their abdomen. In order to evaluate the SAR on this situation, the normal-mode helical antenna (NHA) on the portable radio terminals must be modeled.

In the previous study [6], the SAR calculation (by the Finite-Difference Time-Domain (FDTD) method) and measurement of human body has been investigated by use of the dipole type NHA at $150 \mathrm{MHz}$. However, the portable radio terminals for business is composed of the monopole type NHA and a small radio terminal in general. Therefore, it is important to calcu- 
late and measure the SAR by use of the actual portable radio terminals for business.

In this study, in order to evaluate the SAR of the human body when wearing a portable radio terminal for business, the NHA with metallic case was precisely modeled using $0.1 \mathrm{~mm}$ voxels for the accurate assessment. No studies have yet been precisely modeled the NHA such as this antenna model. In addition, the calculations of SAR distributions employing an NHA with metallic case in the vicinity of the tissue-equivalent phantom were compared with the measurements.

\section{Analytical model}

Fig. 1 (a) shows the NHA with metallic case at $150 \mathrm{MHz}$ used for these calculations. It is composed of the NHA for amateur radio (D90-1018-35, by Kenwood Corporation, Tokyo, Japan) and the metallic case which simulates the amateur radio terminal. Fig. 1(b) shows the analytical model. The distance between the antenna and surface of the phantom is $40.0 \mathrm{~mm}$. The

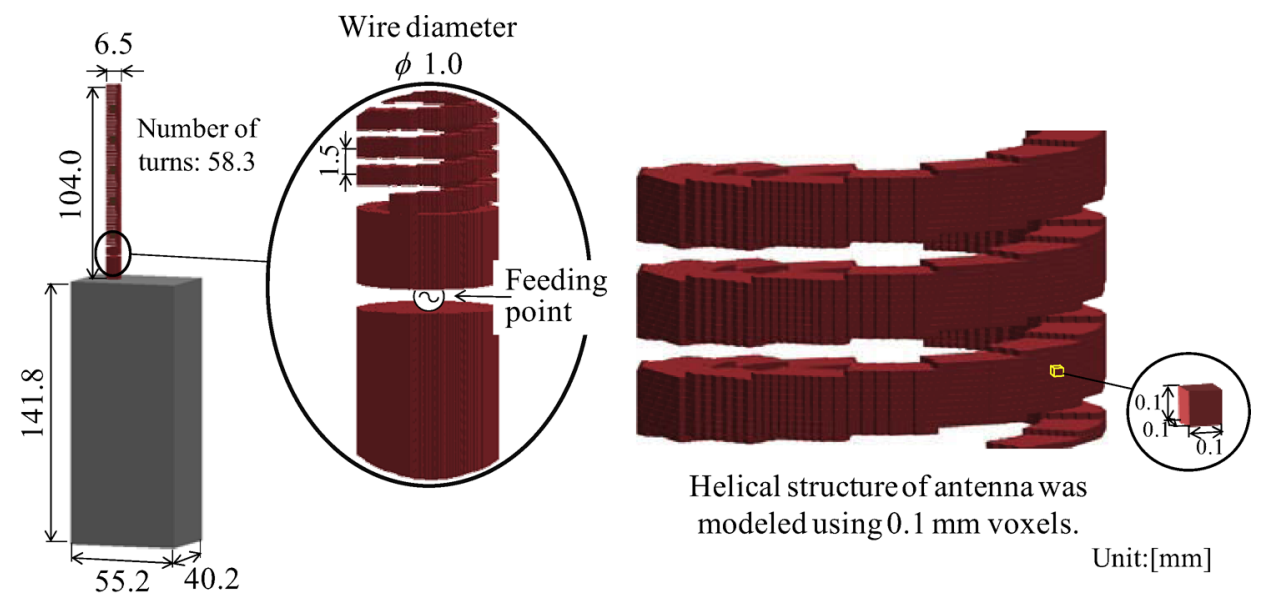

(a)

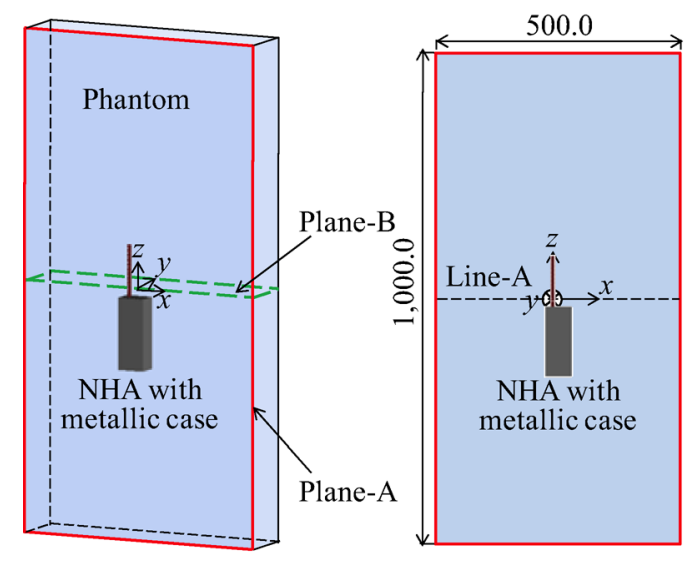

$3 \mathrm{D}$ view
Plane-A

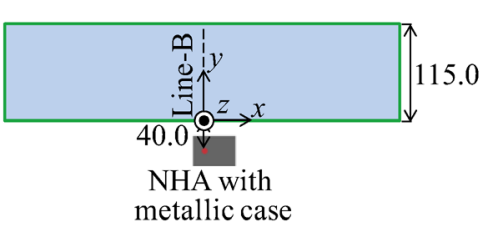

Plane-B

(b)

Fig. 1. NHA with metallic case (a) and analytical model (b). 
observation planes are at the surface of phantom (Plane-A) and the horizontal plane of the phantom including the feeding point of the antenna (Plane-B). In addition, the observation lines are the line passing through the coordinate origin on parallel to the $x$ axis (Line-A) and directly under the feeding point along the $y$ axis on the horizontal plane (Line-B). The coordinate origin is center of surface on the phantom. Here, the target values of the physical properties of the phantom are as follows: relative permittivity $\varepsilon_{r}$ is 52.3 ; conductivity $\sigma$ is $0.76 \mathrm{~S} / \mathrm{m}$; density $\rho$ is $1,030 \mathrm{~kg} / \mathrm{m}^{3}$. These values were taken from [7]. The differences between the values used in this study and the target values were within $5 \%$.

\subsection{Numerical calculation}

In the numerical calculation, the electric fields ( $E$-field) around the antenna are calculated by the FDTD method. This technique is a well-known effective method for the calculation of the SAR as one of the EM analyses. The parameters of the FDTD calculation employed in this study are as follows; the cell size of NHA is $0.1 \mathrm{~mm}$; the phantom to free space is $0.1-2.0 \mathrm{~mm}$. In addition, the helical structure of antenna is precisely modeled using $0.1 \mathrm{~mm}$ voxels (Fig. 1(a)). Here, the coaxial cable used in the measurement is modeled as perfect electric conductor. The absorbing boundary condition is employed the perfectly matched layer (PML) (eight layers). Here, the calculations are performed by own FDTD code.

\subsection{SAR measurement}

Fig. 2 shows the SAR measurement system. The phantom is a flat phantom filled by the tissue-equivalent liquid. In this study, the SAR distribution is measured by the DASY4 system (ver. 4.7, by Schmid \& Partner Engineering AG, Zurich, Switzerland), which employs the E-field probe method [8]. In this method, the SAR is evaluated by Eq. (1) and the measurement of the $E$-field distribution inside the flat phantom, which is composed by a tissueequivalent liquid and a shell, using the isotropic $E$-field probe. This method is generally used for the SAR estimation in the standards [9].

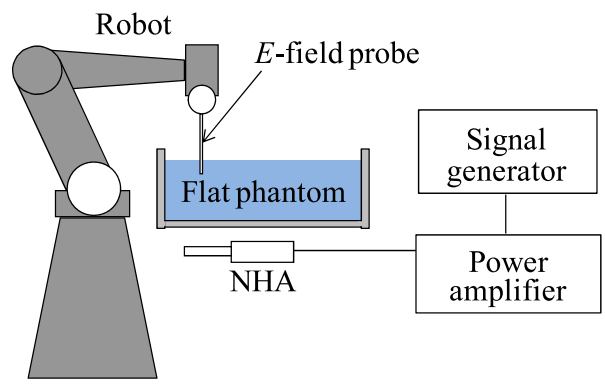

\begin{tabular}{c|c|c}
\hline Device & $\begin{array}{c}\text { Model } \\
\text { number }\end{array}$ & Manufacturer \\
\hline $\begin{array}{c}\text { Signal } \\
\text { generator }\end{array}$ & SMIQ03B & Rohde \& Schwarz \\
\hline $\begin{array}{c}\text { Power } \\
\text { amplifier }\end{array}$ & $100 \mathrm{~W} 1000 \mathrm{~B}$ & AR \\
\hline Robot & RX90L & Stäubli \\
\hline $\begin{array}{c}\text { E-field } \\
\text { probe }\end{array}$ & ET3DV6 & $\begin{array}{c}\text { Schmid \& Partner } \\
\text { Engineering AG }\end{array}$ \\
\hline
\end{tabular}

Fig. 2. SAR measurement system. 


\section{Results}

Figs. $3(\mathrm{a})$ and (b) show the calculated and the measured results of SAR distribution in the observation planes (plane-A and B). Here, the radiated powers of the antennas are normalized to $1.0 \mathrm{~W}$ in both cases. In addition, the SAR distributions of plane-A and B are $4.0 \mathrm{~mm}$ inside the phantom surface because of the limitation of measurement area of $E$-field probe. As shown in Figs. 3 (a) and (b), the SARs at the vicinity of the antenna feeding point are the highest value at both calculated and measured results. In addition, the calculated and measured SARs at surface of the phantom below the metallic case $(z \leq-140 \mathrm{~mm})$ are approximately $0.15 \mathrm{~W} / \mathrm{kg}$. This is attributed to the effect of a coaxial cable for feeding.

Figs. $3(\mathrm{c})$ and (d) show the SAR distributions on the observation line-A and line-B. As shown in Fig. 3 (c), the SAR of calculated result has similar tendency to the measured result on the surface of phantom. Moreover in Fig. 3 (d), two results show similar tendency in deep portion of the phantom. The difference between the calculated and the measured result is within $5 \%$.

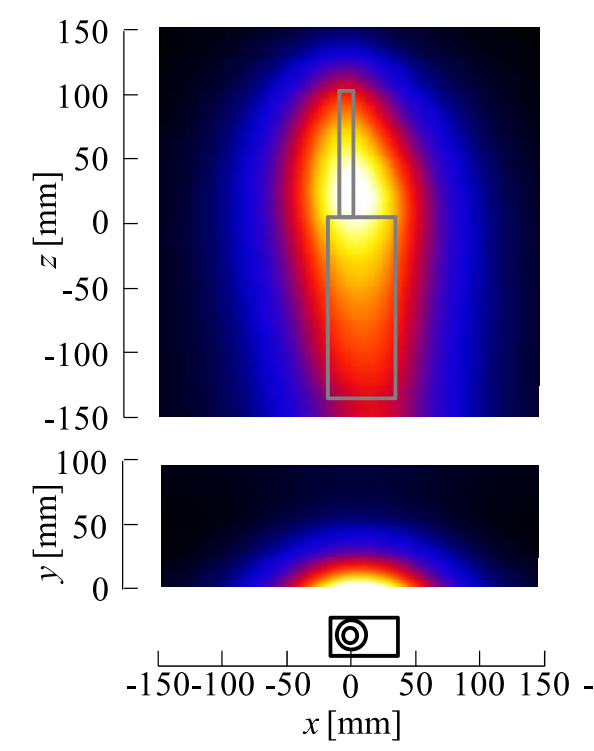

(a)

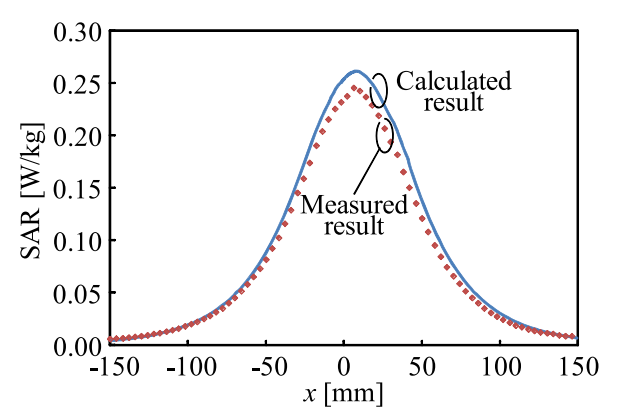

(c)
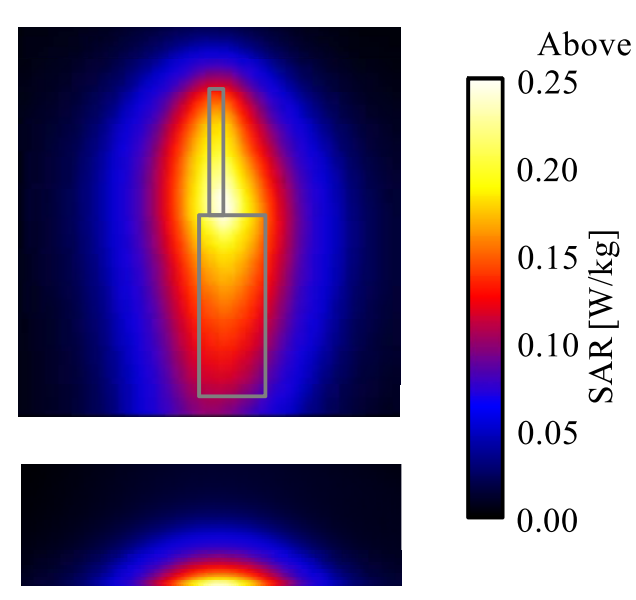

@

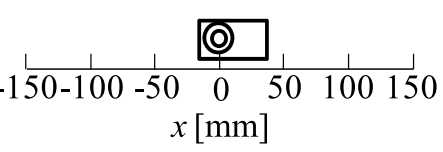

(b)

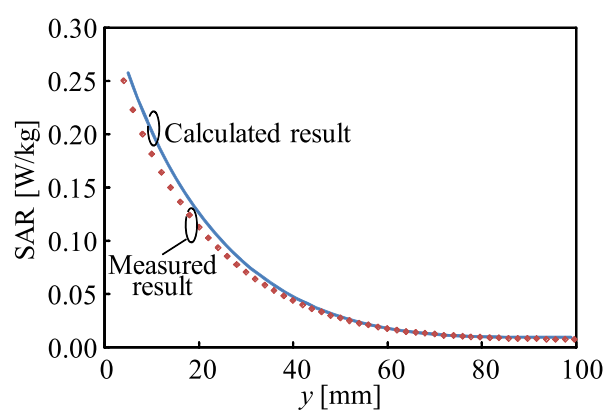

(d)

Fig. 3. The calculated result (a) and the measured result (b) of SAR distributions in the observation planes. The SAR distribution on the observation line-A (c) and on the line-B (d).

(c) IEICE 2009

DOI: 10.1587/elex.6.477

Received February 13, 2009

Accepted February 27, 2009

Published April 25, 2009 
From these results, it has been observed that the measured SAR distributions agree well with calculated results.

\section{Conclusion}

In this paper, the NHA with metallic case was precisely modeled using $0.1 \mathrm{~mm}$ voxels for the accurate estimation. In addition, the calculations of SAR distributions employing an NHA with metallic case in the vicinity of the tissueequivalent phantom were compared with the measurements. As a result, the SAR distributions of calculated result agree well with those of measured result.

\section{Acknowledgments}

The authors would like to thank Kenichi Sato, NTT Advanced Technology Corporation, Tokyo, Japan for his cooperation towards the measurement. In addition, the authors would like to thank Ryotaro Suga, Faculty of Engineering, Chiba University, Chiba, Japan for his cooperation towards the modeling of NHA. Finally, the authors would like to thank Dr. Soichi Watanabe and Dr. Lira Hamada, National Institute of Information and Communications Technology, Tokyo, Japan for their advice concerning this study. 